\title{
Otimização de equipamentos em obras de terraplanagem utilizando programação linear inteira
}

\author{
Bruna de Almeida Morais ${ }^{1}$, Viviane Adriano Falcão ${ }^{2}$ \\ 1Departamento de Engenharia Civil, Instituto de Ciências Tecnológicas e Exatas, UFTM, bruna.alm.morais@gmail.com \\ 2Departamento de Engenharia Civil, Instituto de Ciências Tecnológicas e Exatas, UFTM, viviane.falcao@uftm.edu.br
}

\section{Recebido:}

21 de agosto de 2018

Aceito para publicação:

30 de outubro de 2018

Publicado:

31 de dezembro de 2019

Editor de área:

Francisco Thiago Aragão

\section{Palavras-chaves:}

Terraplanagem,

Problema de Roteamento,

Equipamentos,

Distância Média de Transporte.

\section{Keywords:}

Earthmoving,

Routing Problem,

Equipment,

Average Transport Distance.

DOI:10.14295/transportes.v27i4.1814

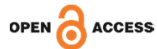

(c) (i)

\begin{abstract}
RESUMO
Com o intuito de reduzir os gastos da terraplanagem, vários estudos abordaram formas de promover a diminuição das distâncias percorridas pelos veículos transportadores, encontrando distâncias de transporte ótimas que atendam às demandas das seções de corte e aterro. Outros buscaram, ainda, encontrar rotas para os veículos de forma que todo o seu itinerário resulte na menor distância total de transporte possível, porém não consideraram a composição da frota de equipamentos e as limitações que cada um deles possui. Este trabalho tem como objetivo reformular o modelo de roteamento para múltiplos veículos de maneira que o intervalo de distâncias de transporte ideal para cada tipo de maquinário seja incluso nele. O novo modelo foi aplicado a um exemplo fictício para fins de validação, e os resultados obtidos demonstram que o modelo é capaz de atender diferentes faixas de distâncias, fornecendo a trajetória ótima para equipamentos diversos.
\end{abstract}

\begin{abstract}
Aiming to reduce earthwork expenses, many studies have approached ways to promote the decrease of the distances traveled by the hauling vehicles, finding optimal haul distances that meet the demand of the cut and fill sections. Others have even searched to find routes for the vehicles, so that their whole itinerary results on the smallest possible haul distance, but they have not considered the composition of the equipment fleet and the limitations each of them have. This work aims to reformulate the multiple vehicle routing model so that the ideal haul distance interval for each type of machinery was included in it. The new model was applied to a fictitious example for the purpose of validation, and the results demonstrated that the model is capable of attending different distance ranges, providing the optimal trajectory for diverse equipment.
\end{abstract}

\section{INTRODUÇÃO}

O desenvolvimento econômico de uma região e a capacidade de seu sistema de transportes estão intrinsecamente relacionados, pois, à medida que este é majorado, comportando maior movimentação de pessoas e cargas, aquele aparece como uma consequência, devido ao aumento das possibilidades de comércio, trabalho e intercâmbio científico e tecnológico. Da mesma forma, à medida que uma região se desenvolve economicamente, é necessária a adaptação do sistema de transportes à sua expansão e à nova demanda de deslocamento. Assim, o estudo dos sistemas de transporte e obras de infraestrutura viária é recomendável ao planejamento do progresso de uma localidade.

No Brasil, o modal rodoviário tem expressiva participação no transporte de carga como um todo, o que indica a necessidade de haver a adequação de suas vias ao grande volume de trans- 
porte a ser realizado, através de diversas obras rodoviárias de infraestrutura, seja para implementação, ampliação ou manutenção de rodovias. Como os valores despendidos nessas obras são altos, o que se justifica por sua extensão e sua frequência, a minimização das despesas em quaisquer de suas fases tem como resultado uma redução significativa no seu valor total, principalmente se for uma etapa com participação expressiva no custo da obra, como a terraplanagem.

Considerando-se que a terraplanagem corresponde a cerca de $25 \%$ do custo total da obra rodoviária, podendo esse percentual ser bem maior em obras com alta dificuldade de execução (HARE et al., 2011), sua otimização tem um grande impacto no custo final da obra como um todo. Ainda de acordo com Hare et al. (2011), um valor preciso do custo ótimo de terraplanagem é essencial para a avaliação de um determinado projeto de rodovia. Assim, para obtenção do custo ótimo, é preciso determinar as rotas entre corte e aterro de forma que o transporte de terra seja o mínimo, além da utilização do equipamento mais adequado de acordo com as características da obra.

Alguns modelos matemáticos, como o de Mayer e Stark (1981), Easa $(1987,1988)$ e Jayawardane e Harris (1990), foram desenvolvidos buscando minimizar o custo das operações de terraplanagem, mas grande parte destes não observa como o percurso dos equipamentos utilizados poderia impactar na diminuição dos custos. Já outros, como de Henderson et al (2003), buscam encontrar a rota percorrida pelos veículos que resulte no menor custo. Porém, no estudo mais recente a considerar esse roteamento, de Henderson et al (2003), a variável da distância de transporte não foi examinada como um fator determinante para a escolha do maquinário a ser utilizado.

A inclusão da variável distância de transporte no modelo de otimização poderia resultar em um modelo que represente melhor a realidade em uma operação de terraplanagem, e, consequentemente, pode ser uma forma de determinação do equipamento mais econômico a ser utilizado. Segundo Day e Benjamin (1991), os scrapers são os equipamentos mais indicados para operações de corte e aterro para valores de distância de transporte entre 300 pés, cerca de 92 metros, e 1,5 milha, cerca de 2400 metros. Porém, os autores referem-se a scrapers de maneira ampla, não especificando se são motoscrapers ou scrapers rebocados, os quais têm capacidade de percorrer distâncias mais extensas.

Ainda conforme Day e Benjamin (1991), para distâncias abaixo de 300 pés, o custo de utilização de um scraper deve ser comparado ao custo de utilização de um dolzer. Já para distâncias de transporte longas, essa comparação deve ser feita com escavadeiras ou pás carregadeiras e unidades transportadoras. Além do valor da distância de transporte, a escolha do equipamento a ser utilizado deve ser feita considerando-se outros fatores, como as características do solo a ser escavado, a declividade do terreno e o espaço disponível para manejo e condução do maquinário, entre outras. Apesar disso, Ricardo e Catalani (2007) afirmam que, se considerado individualmente, o valor da distância de transporte é o principal fator a ser considerado na escolha do equipamento.

Segundo esses autores, para distâncias consideradas pequenas, menores que 50 metros, é indicado o uso de máquinas de esteira com lâmina, que, apesar de apresentarem baixas velocidades, inferiores a $10 \mathrm{~km} / \mathrm{h}$, apresentam também custos baixos. Já para distâncias maiores que $100 \mathrm{~m}$, os autores recomendam o uso de scrapers rebocados ou motoscrapers, que transportam maiores volumes. 
Ricardo e Catalani (2007) dizem, ainda, que de acordo com estudos feitos pelo Highway Research Board, quando as distâncias de transporte compreendem-se entre 50 metros e 200 metros, o scraper rebocado é utilizado mais frequentemente. Já o motoscraper, se for pequeno ou médio, é preferido na faixa de 100 metros a 400 metros, e, se for de grande capacidade ou de dois motores, são indicados para distâncias entre 300 metros e 750 metros. Por outro lado, para distâncias superiores a 900 metros, os autores recomendam a adoção de equipamentos de baixo custo, viabilizando uma frota com muitas unidades de transporte e carregamento realizado por máquinas escavocarregadoras.

Assim, o objetivo deste trabalho é propor um modelo que considere o tipo de maquinário a ser utilizado nas operações de terraplanagem como um fator limitante das distâncias a serem percorridas e seu consequente reflexo no custo total e no tempo gasto para realizar essas operações.

\section{MODELOS MATEMÁTICOS APLICADOS À TERRAPLANAGEM}

Nos modelos que foram previamente aplicados à terraplanagem, existem duas abordagens distintas. A primeira diz respeito ao problema de transporte, que visa à redução do custo total das viagens, sem analisar qual rota o caminhão deve percorrer. Assim, o resultado fornecido é apenas de quais seções de excesso e para quais seções de déficit a terra deve ser levada, sem considerar o custo do transporte que decorrerá dos deslocamentos dos veículos nos trechos intermediários entre essas seções. A segunda diz respeito ao problema de roteamento, que fornece, como resultado, todo o caminho ordenado a ser percorrido pelos veículos durante as operações de terraplanagem, oferecendo um cálculo de custo total mais próximo do real.

\subsection{Modelos matemáticos aplicados ao problema de transporte na terraplanagem}

Mayer e Stark (1981) formularam um modelo em que o custo total deve ser minimizado, determinando-se os valores das variáveis de distância. Algumas seções possuem determinados volumes de solo disponíveis para corte, e outras devem ser aterradas. Assim, o modelo visa a determinar quanto do solo disponível para corte em cada seção deve ser transportado para as seções a serem aterradas.

O custo unitário é determinado de acordo com o custo de escavação e aterramento de cada um desses locais e o custo de transporte, sendo este último resultante da distância entre eles e do custo por unidade de distância percorrida. 0 custo total será a soma dos valores das multiplicações de cada volume a ser transportado de um local de corte a um local de aterro pelo custo unitário de cada uma dessas operações de um local a outro.

Esses autores consideraram no modelo as variações que o empolamento e a compactação do solo nesses locais também ensejam nos valores de custo unitário, uma vez que o volume de solo tende a aumentar quando escavado e a diminuir quando compactado. Outro fator que foi considerado pelos autores é a necessidade ou a conveniência de serem estabelecidos locais de disposição ou de empréstimo nas proximidades da rodovia, o que geraria custos de implantação, como a aquisição do terreno ou obtenção de servidão de passagem, a preparação do terreno para escavação ou aterramento e a construção e manutenção de vias de acesso. Assim, o modelo fornece também quais são os volumes a serem transportados para esses locais de disposição ou recebidos desses locais de empréstimos, caso sejam utilizados.

Easa $(1987,1988)$ expandiu o modelo de Mayer e Stark, de forma que este pudesse abranger variáveis de custo lineares e não constantes, já que diversos fatores podem fazer com que os 
custos unitários variem. 0 autor exemplifica esses fatores, abordando variações nos custos unitários de escavação, que dependem da quantidade de material a ser escavado e do tipo de solo, variações nos custos unitários de transporte, que dependem, entre outros condicionantes, dos tipos de unidades transportadoras, da distância de transporte e da oscilação de preços de acordo com a faixa de volume a ser transportado, e variações no custo unitário de compactação, que é determinado de acordo com o tipo de equipamento a ser utilizado, este escolhido conforme o tipo de solo, o nível de compactação requerido e outras condições operacionais.

0 autor também aborda a variação nos custos de aquisição de áreas de empréstimo, que mudam de acordo com a localização da área, se em zona rural ou urbana, e que podem mudar de acordo com a quantidade utilizada, e de áreas de bota-fora, que representam uma porção muito pequena quando comparados aos custos de outras atividades de terraplanagem, uma vez que, na maioria das vezes, esse custo não varia conforma a quantidade de material a ser disposto.

Easa $(1987,1988)$ analisa, portanto, quais os fatores que variam de acordo com a quantidade de material a ser operado, reformulando-os como variáveis não constantes, sendo esses fatores o custo de aquisição e de escavação em uma área de empréstimo. As outras variáveis, que não dependiam da quantidade de material, foram inseridas no modelo como constantes. Assim, o autor tornou o modelo mais adequado à realidade das atividades de terraplanagem.

Jayawardane e Harris (1990) incorporaram ao modelo de Mayer e Stark (1981) a duração do projeto de acordo com as distâncias de transporte e outros fatores práticos, como a variação de extratos do solo, diferentes graus de compactação em extratos diferentes de um mesmo solo, um número maior de áreas de empréstimo e locais de disposição, além de condicionais de tempo, como a necessidade de que uma seção da rodovia seja concluída antes que as obras comecem em outra.

\subsection{Modelos matemáticos aplicados ao problema de roteamento na terraplanagem}

Henderson et al. (2003) abordaram o Problema do Caminho Mínimo entre Corte e Aterro (Shortest Route Cut and Fill Problem - SRCFP), que busca uma rota para um único veículo transportador de solo que minimize a distância total percorrida entre localizações de corte e aterro. Esse problema baseia-se no Problema do Caixeiro Viajante - PCV, o qual consiste em encontrar um passeio através de um circuito hamiltoniano (ou seja, um circuito em que todos os vértices devem ser visitados apenas uma vez e em que se deve retornar ao vértice inicial) em que cada um de seus vértices representa uma cidade a ser visitada pelo caixeiro (GOLDBARG; LUNA, 2005).

O SRCFP diferencia-se do PCV pelo fato de que o veículo deve visitar, alternadamente, vértices que representam seções de corte e vértices que representam seções de aterro, além da possibilidade de um local ser visitado mais de uma vez (LIM; RODRIGUES; ZHANG, 2005).

Como a enumeração de todas as possibilidades seria um processo ineficiente, considerandose o grande número de seções, Henderson et al. desenvolveram um algoritmo que pudesse fornecer a rota ótima ou quase ótima para um único veículo. Para desenvolvê-lo, esses autores determinaram que uma rota é o caminho percorrido pelo veículo de forma a visitar todas as seções de corte e aterro, alternadamente, uma única vez, e retornar à seção de corte inicial. 0 parâmetro a ser minimizado é a distância total de transporte, que corresponde à extensão de uma rota.

Assim, ainda segundo Henderson et al. (2003), o problema de roteamento difere do problema de transporte ao considerar a necessidade de que o veículo atenda alternadamente a uma seção de corte e uma de aterro, buscando minimizar, assim, o custo total resultante das viagens entre seções de corte e aterro, e não apenas o custo do percurso entre seções. Os autores utilizaram 
um algoritmo de recozimento simulado e modelaram o SRCFP como um problema de otimização discreta.

Falcão (2016) aumentou a abrangência do modelo ao reformulá-lo de forma a obter a solução do Problema do Caminho Mínimo entre Corte e Aterro para múltiplos caminhões, problema conhecido como Problema de Roteamento de Corte e Aterro com Múltiplos Caminhões (Truck Route Cut and Fill Problem - TRCFP). Dessa forma, o modelo torna-se aplicável a um número maior de operações de terraplanagem, que utilizam mais de um veículo transportador, o que apresenta como vantagem a possibilidade de uma redução drástica no tempo total de operação. Assim, o modelo pode fornecer as rotas mais econômicas para cada caminhão basculante, de forma a obter-se um itinerário otimizado da operação de terraplanagem.

Assim, o modelo da autora viabiliza a definição das rotas ótimas para cada caminhão de forma a minimizar a distância total de transporte percorrida por cada um deles, e, consequentemente, a distância total de transporte de toda a operação de terraplanagem. Apesar disso, o modelo não contempla a possibilidade de que um tipo de unidade transportadora possa ser mais viável que outras, conforme essa distância total percorrida. Assim, a inclusão da restrição referente à DMT nesse modelo poderia resultar em uma escolha de equipamento mais eficiente.

\section{PROBLEMA DE ROTEAMENTO COM MÚLTIPLOS VEÍCULOS COM RESTRIÇÃO DE DISTÂNCIA DE TRANSPORTE}

Inicialmente, foram definidos os fundamentos do problema para possibilitar sua devida compreensão. Em seguida, o SRCFP proposto por Henderson et al. (2003), com um único veículo de transporte, foi formulado computacionalmente, assim como o TRCFP proposto por Falcão (2016). Assim, foi possível estabelecer as bases para a inserção de uma nova restrição no modelo.

Por fim, a limitação referente à Distância de Transporte foi incorporada ao modelo do TRCFP, e foi feita a validação deste novo modelo através de um exemplo. Esse exemplo foi, também, aplicado nos modelos de SCRFP e TRCFP para fins de comparação.

\subsection{Definição do problema}

O Problema de Roteamento de Corte e Aterro com Múltiplos Caminhões mostra-se eficiente quanto à determinação do fluxo a ser percorrido pelos veículos em obras rodoviárias de terraplanagem. Apesar disso, sabe-se que as distâncias a serem percorridas por esses veículos variam de acordo com as limitações do equipamento escolhido, e que a utilização do maquinário inadequado pode ter consequências impactantes no custo total da obra. Assim, ao decidir previamente qual deve ser o tipo de equipamento utilizado, ou qual equipamento deve ser evitado, é possível limitar as rotas a serem percorridas pelos veículos para que suas extensões não sejam superiores ou inferiores à faixa ótima de utilização do maquinário.

Assim, se a análise das condições da obra estabelecer que, por algum fator natural, técnico ou operacional, um determinado equipamento não pode ser utilizado, a faixa ideal de distâncias na qual esse equipamento atua pode ser excluída no modelo. Dessa forma, o veículo escolhido para realizar as operações de terraplanagem, que terá um intervalo de distâncias ideais distinto, não percorrerá trechos com extensões na faixa de atuação do equipamento descartado. 


\subsection{Formulação matemática}

O modelo do problema de roteamento com múltiplos veículos e restrição de distância de transporte é representado nas Equações (1) a (11).

Sujeito a:

$$
\text { Minimizar } Z=\sum_{(i, j) \in A} D_{i j} X_{i j k}
$$

$$
\begin{gathered}
\mathrm{x}_{\mathrm{ijk}}+\mathrm{x}_{\mathrm{jik}} \leq 1 \quad \forall(\mathrm{i}, \mathrm{j}) \in \mathrm{A}, \text { com } \mathrm{i}<\mathrm{j} \\
\sum_{\mathrm{i} \neq \mathrm{j}} \mathrm{f}_{\mathrm{ij}}-\sum_{\mathrm{i} \neq \mathrm{j}} \mathrm{f}_{\mathrm{ji}}=1, \forall \mathrm{i}, \mathrm{j} \in \mathrm{G} / \mathrm{j} \neq 1, \mathrm{i}, \mathrm{j}=1, \ldots, \mathrm{p} \\
\mathrm{f}_{\mathrm{ij}} \leq(\mathrm{n}-1) \mathrm{x}_{\mathrm{ijk}}, \forall \mathrm{i}, \mathrm{j} \in \mathrm{G} \\
\mathrm{x}_{\mathrm{ijk}}+\mathrm{x}_{\mathrm{jik}}=0, \forall(\mathrm{i}, \mathrm{j}) \in \mathrm{A} / \mathrm{DT}_{\text {mín }} \geq \mathrm{d}_{\mathrm{ij}} \geq \mathrm{DT}_{\text {máx }}, \mathrm{d}_{\mathrm{ij}} \in \mathrm{D} \\
\sum_{\mathrm{j}} \mathrm{x}_{\mathrm{ijk}}=\mathrm{y}_{\mathrm{ik}}, \mathrm{i}=1, \ldots, \mathrm{m} \text { e } \mathrm{k}=1, \ldots, \mathrm{t} \\
\sum_{\mathrm{j}} \mathrm{x}_{\mathrm{ijk}}=\mathrm{y}_{\mathrm{jk}}, \mathrm{j}=1, \ldots, \mathrm{n} \text { e k }=1, \ldots, \mathrm{t} \\
\sum_{\mathrm{k}} \mathrm{y}_{\mathrm{ik}}=1, \mathrm{i}=1, . ., \mathrm{n} \\
\sum_{\mathrm{i}, \mathrm{j}} \mathrm{x}_{\mathrm{ijk}} \leq \mathrm{v}, \mathrm{k}=1, \ldots, \mathrm{t} \\
\mathrm{x}_{\mathrm{ijk}} \in\{0,1\} \quad \forall(\mathrm{i}, \mathrm{j}) \in \mathrm{A} \mathrm{e} \mathrm{k}=1, \ldots, \mathrm{t} \\
\mathrm{y}_{\mathrm{jk}} \in\{0,1\} \mathrm{j}=1, \ldots, \mathrm{n} \text { e k }=1, \ldots, \mathrm{t}
\end{gathered}
$$

em que $\quad \mathrm{m}$ : número de unidades de corte;

n: $\quad$ número de unidades de aterro;

$\mathrm{w}$ : $\quad$ quantidade de unidades de corte e aterro, ou seja $\mathrm{m}+\mathrm{n}$;

A: $\quad$ conjunto de arcos de cortes para os aterros e de aterros para cortes;

p: $\quad$ quantidade de seções de corte ou aterro,

G vetor de posições das seções de corte e aterro;

$\mathrm{K}$ : $\quad$ quantidade de caminhões, $\mathrm{k}=1,2, \ldots, \mathrm{t}$;

$\mathrm{D}_{\mathrm{ij}}$ : $\quad$ matriz de distâncias entre seções de corte e aterro;

Xijk: $\quad$ variável binária que assumo o valor 1 quando o caminha k transporta material de corte i para o aterro j, sendo 0 , caso contrário;

yik: $\quad$ variável binária que assume o valor 1 quando o caminhão k transporta material de corte $i$, sendo 0 , caso contrário;

$\mathrm{f}_{\mathrm{ij}}$ : $\quad$ variável de fluxo que assume o valor positivo $\mathrm{s}$ e $\mathrm{x}_{\mathrm{ijk}}=1, \mathrm{f}_{\mathrm{ijk}} \in \mathrm{R}^{+}$;

DT: a distância de transporte limite do equipamento, a depender do seu tipo, sendo DT mín a distância mínima a ser percorrido e DT $\mathrm{max}_{\text {ax }}$ distância máxima a ser percorrida;

v: $\quad$ variável de balanceamento de veículos, sendo determinada de acordo com a proporcionalidade desejada.

A função objetivo (1) minimiza a distância total percorrida pelo veículo que faz o transporte de terra entre seções de corte e de aterro, alternadamente. A restrição (2) impede a formação 
de subcircuitos, ou seja, impede que o veículo retorne a uma seção intermediária de sua rota sem atender todas as seções. As restrições (3) e (4) têm como objetivo garantir a continuidade do fluxo, assegurando que o caminhão siga para a próxima seção.

O conjunto de restrições (6) e (7) assegura que de uma seção de corte o veículo deve seguir para uma única seção de aterro, impedindo que o percurso seja interrompido, enquanto a restrição (8) garante que uma unidade de corte ou aterro será atendida por uma única máquina. Já a restrição (9) tem como objetivo evitar a sobrecarga de veículos em cada trecho e, consequentemente, uniformizar a distribuição de viagens para o maquinário disponível.

Por fim, a restrição (5) limita distância de transporte a ser percorrida em cada trecho de acordo com o equipamento a ser utilizado. Ao serem determinadas as distâncias mínima e máxima do maquinário, ou seja, sua faixa de atuação, o modelo exclui do percurso os trechos que não se encontrem nesse intervalo, adequando as operações ao desempenho do equipamento.

\subsection{Validação do modelo}

Para validar o modelo, foi utilizado um exemplo fictício de um trecho de estrada com nove seções de corte e nove de aterro, com diferentes volumes e distâncias entre si. 0 objetivo desse exemplo foi possibilitar que um mesmo trecho comportasse distâncias de diversos valores entre as seções, de forma que pudessem ser adotados múltiplos tipos de equipamentos com os quais fosse possível encontrar soluções. A Tabela 1 apresenta as distâncias, em metros, entre os centros de massa de cada seção de corte a cada seção de aterro.

Tabela 1 - Distâncias, em metros, entre as seções de corte e aterro do exemplo fictício

\begin{tabular}{ccccccccccc}
\hline DISTÂNCIAS & \multicolumn{10}{c}{ ATERROS } \\
\hline & & 1 & 2 & 3 & 4 & 5 & 6 & 7 & 8 & 9 \\
\cline { 2 - 10 } & 1 & 350 & 700 & 850 & 1000 & 1200 & 1300 & 1600 & 1600 & 1800 \\
& 2 & 500 & 450 & 600 & 800 & 1000 & 1100 & 1300 & 1200 & 1500 \\
CORTES & 3 & 550 & 400 & 600 & 650 & 800 & 850 & 1000 & 950 & 1050 \\
& 4 & 450 & 300 & 750 & 700 & 700 & 850 & 1100 & 1000 & 1500 \\
& 5 & 850 & 650 & 600 & 650 & 550 & 600 & 650 & 950 & 1150 \\
& 6 & 950 & 800 & 500 & 600 & 400 & 700 & 550 & 750 & 1000 \\
& 7 & 1400 & 1200 & 1100 & 1250 & 500 & 350 & 450 & 350 & 750 \\
& 8 & 1450 & 1250 & 900 & 1100 & 700 & 450 & 500 & 550 & 750 \\
& 9 & 1650 & 1600 & 950 & 1350 & 1200 & 450 & 750 & 600 & 850 \\
\hline
\end{tabular}

Quanto aos volumes de corte, considerou-se que os Cortes 1, 3, 4, 6, 7 e 9 requeriam a retirada de $4 \mathrm{~m}^{3}$ de terra, enquanto os Cortes 2, 5 e 8 requeriam a retirada de $3 \mathrm{~m}^{3}$. Já quanto aos volumes de aterro, considerou-se que os Aterros 1, 4 e 7 comportavam $5 \mathrm{~m}^{3}$, enquanto os Aterros 2, 3, 5, 6, 8 e 9 comportavam $3 \mathrm{~m}^{3}$. Para encontrar a quantidade de unidades de cortes ou de aterro em cada um dos pontos analisados, foram divididos seus volumes totais pelo valor da unidade de carregamento, ou seja, a capacidade do veículo, que foi adotada como $1 \mathrm{~m}^{3}$, independentemente do seu tipo.

Para a solução computacional do problema, foi utilizado o CPLEX 12.6, que é um software da IBM que oferece bibliotecas C, C++, Java, .NET e Python que resolvem programação linear e problemas relacionados em que o objetivo a ser otimizado pode ser expresso como uma função linear (IBM, 2014). 0 programa foi executado em um sistema operacional Windows 10 de 64 Bits, com processador Intel Core i7 e 8 GB de memória RAM.

Inicialmente, para fins de comparação, o exemplo foi aplicado no modelo SRCFP. A distância 
de transporte total obtida foi de 34800 metros. Em seguida, foi testado o modelo TRCFP, para dois caminhões, e a distância de transporte obtida foi de 34750 metros. Posteriormente, o exemplo foi utilizado para a validação do modelo com a limitação de distância de transporte. Novamente, foram adotados dois veículos com capacidade de $1 \mathrm{~m}^{3}$, prezando a facilidade de compreensão do exemplo. Foram consideradas duas situações: uma em que uma equipe composta por motoscrapers e pushers não é aceitável e outra em que uma equipe composta por carregadeiras ou escavadeiras e caminhões basculantes não pode ser utilizada.

Na primeira situação, as distâncias de transporte compreendidas na faixa de atuação de um motoscraper devem ser desconsideradas, pois o maquinário a ser utilizado deverá trabalhar com distâncias superiores à distância máxima na qual o motoscraper é viável. Assim, o limite DT mín do modelo foi estabelecido como 750 metros, seguindo as orientações de Ricardo e Catalani (2007), não havendo um limite máximo. Dessa forma, todos os trechos que possuíam extensões inferiores a esse valor foram excluídos das rotas dos veículos, sendo a rota ótima determinada dentre os trechos restantes. Os resultados forneceram uma distância total de transporte de 68000 metros.

Por fim, o modelo foi aplicado à segunda situação, em que não se pode utilizar a combinação de unidades escavocarregadoras e unidades de transporte. Como, segundo Ricardo e Catalani (2007), essa equipe é indicada para distâncias superiores a 900 metros, o limite de distância de transporte utilizado foi DTmáx $=900$ metros. Não foi estabelecido um limite mínimo. Dessa vez, os trechos que possuíam extensões superiores a 900 metros não foram abarcados na rota ótima. A distância total de transporte obtida foi de 35500 metros. Os resultados dos quatro modelos são apresentados na forma de gráfico na Figura 1.

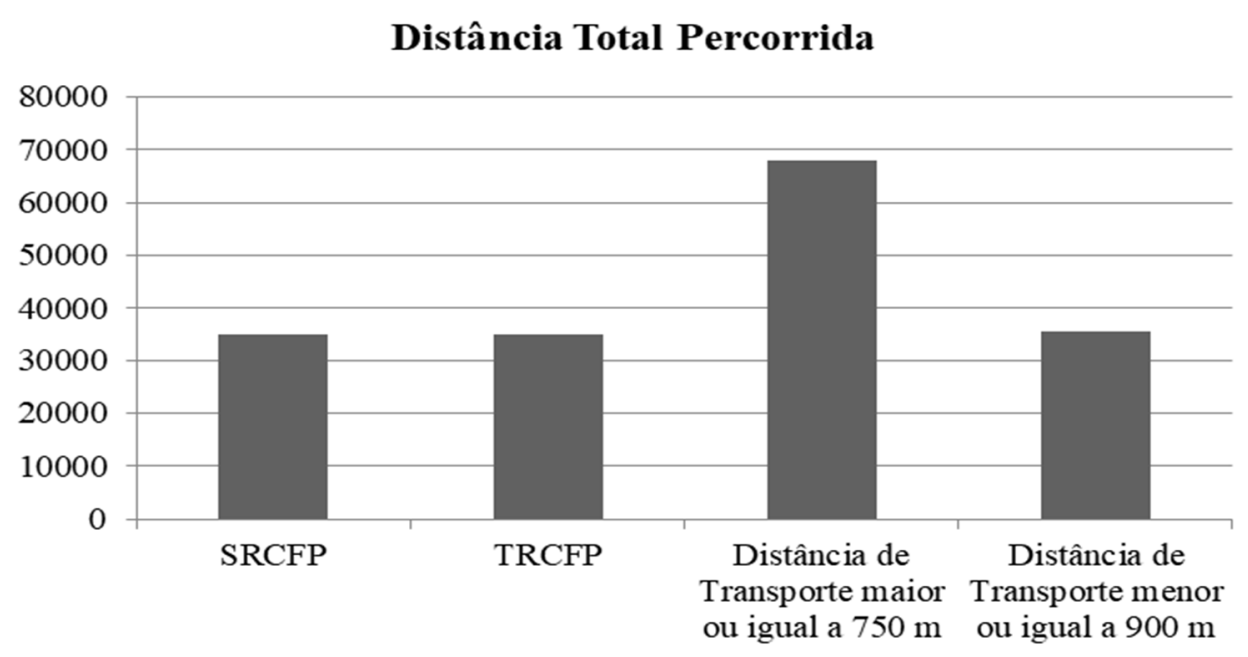

Figura 1. Distância total percorrida em cada modelo

\subsection{Resultados e discussões}

Através dos resultados obtidos, pode-se observar que a distância total de transporte do modelo TRCFP, com dois veículos, foi inferior ao total percorrido por um único caminhão no modelo SRCFP. Observou-se, ainda, que, devido à restrição do modelo que evita a sobrecarga de veículos, o Veículo 1 fez cerca de 55\% das viagens e o Veículo 2, cerca de 45\%, o que indica que o tempo decorrido nas operações de transporte foi reduzido a quase metade do tempo de um 
único veículo. Nota-se, portanto, que a utilização de mais de um veículo teve resultados positivos no exemplo adotado.

Observa-se, também, que a distância total de transporte obtida no modelo com distâncias superiores a 750 metros foi altíssima, quase duas vezes o valor da distância do modelo com distâncias inferiores a 900 metros. A utilização de dois veículos promove uma grande redução no tempo total das operações, se comparado a um único veículo, uma vez que o Veículo 1 fez cerca de $52 \%$ das viagens, e o Veículo 2, cerca de $48 \%$.

Já a distância total de transporte obtida no modelo para a segunda situação foi muito próxima à fornecida pelo modelo TRCFP, que é a distância proveniente da menor rota genérica, ou seja, sem nenhuma especificação quanto ao maquinário e, consequentemente, à distância de transporte. A proporcionalidade de viagens entre os veículos foi semelhante à do TRCFP (Veículo $1=$ $55 \%$ e Veículo $2=45 \%$ ), o que indica que a redução do tempo em relação a um único veículo será a mesma. Assim, percebe-se que equipes que têm seu intervalo de atuação ótimo inferiores a 900 metros são adequadas ao trabalho nas operações de terraplanagem do exemplo adotado, pois, apesar de suas limitações, têm um desempenho próximo ao ideal calculado.

De acordo com as especificações de itens constantes do catálogo da Caterpillar, 2016, as velocidades máximas de um trator-escrêiper carregado e de um caminhão articulado carregado giram em torno de $53.5 \mathrm{~km} / \mathrm{h}$ e $56 \mathrm{~km} / \mathrm{h}$, respectivamente. Essas velocidades foram adotadas como as velocidades médias de operação das equipes nas duas situações de aplicação do exemplo, para fins de cálculo do tempo dispendido no transporte na terraplanagem.

Na primeira situação, como não seria possível utilizar um motoscraper, poderiam ser utilizadas unidades de transporte, como caminhões articulados. Considerando-se que os dois caminhões trabalharam concomitantemente, foram divididas as distâncias percorridas por cada um deles pela velocidade adotada, sendo, assim, obtidos os tempos que levaram para circular todo o seu percurso. Os resultados são apresentados na Tabela 2:

Tabela 2 - Distância e tempo para cada caminhão articulado

\begin{tabular}{llll}
\hline Veículo & Velocidade (km/h) & Distância (metros) & Tempo (minutos) \\
\hline Caminhão 1 & 56 & 36800 & 39,43 \\
Caminhão 2 & 56 & 31200 & 33,43 \\
\hline
\end{tabular}

Na segunda situação, não podendo ser utilizadas unidades de transporte, pode-se optar pelo uso de tratores-escrêiperes. Novamente, foi considerado que os dois tratores-escrêiperes realizaram o trabalho concomitantemente, e o mesmo processo foi utilizado para encontrar os tempos de percurso de cada um deles, mostrados na Tabela 3:

Tabela 3 - Distância e tempo para cada trator-escrêiper

\begin{tabular}{llll}
\hline Veículo & Velocidade (km/h) & Distância (metros) & Tempo (minutos) \\
\hline Trator-escrêiper 1 & 53,5 & 19300 & 21,64 \\
Trator-escrêiper 2 & 53,5 & 16200 & 18,17 \\
\hline
\end{tabular}

O tempo total de operação da primeira equipe será de 21,64 minutos, enquanto o da segunda equipe será de 39,43 minutos. A partir desses resultados, observa-se que o tempo operacional na primeira situação será muito maior do que na segunda, pois as extensões dos trechos a serem percorridos nesta são muito menores do que naquela, e as velocidades médias dos diferentes 
equipamentos são muito próximas. Assim, analisando-se apenas os aspectos das distâncias e do tempo, sem considerar outros fatores que podem influenciar no desempenho do maquinário, a equipe de unidades de transporte deveria ser utilizada apenas em casos extremos, em que não haja outra opção devido à inviabilidade do uso de outras equipes.

\section{CONCLUSÕES}

A adição da limitação da distância de transporte ao modelo apresentou, como benefício, a possibilidade de melhor adequação do modelo a uma situação real, na qual, frequentemente, uma única equipe de maquinário realizará um grande número de operações, não sendo viável que esta percorra distâncias muito variadas. 0 modelo permite, também, uma análise da melhor equipe quando não há um fator limitante, e o critério de decisão é, portanto, exclusivamente econômico ou relativo ao tempo de execução, auxiliando a escolha da composição da equipe de terraplanagem.

Apesar disso, o modelo apresenta limitações pertinentes à frota utilizada. Presume-se que as frotas sejam homogêneas em ambas as situações em que o exemplo foi aplicado, mas pode haver situações reais em que ela não o seja. 0 modelo apresentado neste trabalho não é capaz de atender essas condições, uma vez que não seria possível inserir múltiplos intervalos de distâncias de transporte, pois estas seriam consideradas válidas para todos os veículos, sem diferenciálos.

Assim, para estudos futuros, sugere-se a elaboração de um modelo que seja capaz de atender frotas heterogêneas, além das homogêneas. Outra possibilidade de estudo seria o desenvolvimento de um modelo que aprimore ainda mais o problema do roteamento ao considerar outros fatores que influenciam na decisão dos equipamentos de terraplanagem, como a inclinação do terreno e a capacidade de suporte do solo, tornando-o mais apropriado a uma situação real.

\section{REFERÊNCIAS}

CATERPILLAR. Disponível em: http://www.cat.com/pt_BR/products/new/equipment.html>. Acesso em 05/06/2016.

DAY, D. A. e BENJAMIN, N. B. H. (1991) Construction Equipment Guide. $2^{\text {a }}$ ed. John Wiley \& Sons, Inc. 464 p. (Wiley series of practical construction guides).

EASA, S. (1987). Earthwork Allocations with Nonconstant Unit Costs. Journal of Construction Engineering and Management. 113, 34-50. DOI: 10.1061/(ASCE)0733-9364(1987)113:1(34)

EASA, S. (1988). Earthwork Allocations with Linear Unit Costs. Journal of Construction Engineering and Management. 114(4), 641-655. DOI: 10.1061/(ASCE)0733-9364(1988)114:4(641)

FALCÃO, V. A. (2016) Modelo de roteirização para a terraplenagem em obras rodoviárias aplicando programação linear inteira. 104 f. Tese (Doutorado em Engenharia de Transportes) - Departamento de Engenharia de Transportes, Universidade Federal do Ceará, Fortaleza. 2016.

FALCÃO, V. A.; PRATA, B. A.; NOBRE JÚNIOR, E. F. (2016) Modelo de roteirização para a distribuição de materiais de terraplenagem baseado em programação inteira. Journal of Transport Literature. 10(3), 20-24. DOI: 10.1590/22381031.jtl.v10n3a4

GOLDBARG, M. C.; LUNA, H. P. L. (2005) Otimização Combinatória e Programação Linear. 2 ed. Rio de Janeiro: Campus.

HARE, L. W.; KOCH, V. R.; LUCET, Y. (2011) Models and algorithms to improve earthwork operations in road design using mixed integer linear programming. European Journal of Operational Research. 215, 470-480. DOI: 10.1016/j.ejor.2011.06.011

HENDERSON, et al. (2003) Solving the shortest route cut and fill problem using simulated annealing. European Journal of Operational Research. 145, 72-84. DOI: 10.1016/S0377-2217(02)00206-0

IBM ILOG CPLEX Optimization Studio CPLEX User's Manual (2014). Version 12 Release 6. Disponível em: https://www.ibm.com/support/knowledgecenter/SSSA5P_12.6.1/ilog.odms.studio.help/pdf/usrcplex.pdf>.

JAYAWARDANE, A. K. W.; HARRIS, F. C. (1990) Further development of integer programming in earthwork optimization. Journal of Construction Engineering and Management. 116, 18-34. DOI: 10.1061/(ASCE)0733-9364(1990)116:1(18)

LIM, A; RODRIGUES, B and ZHANG, J. (2005). Tabu search embedded simulated annealing for the shortest route cut and fill problem. Journal of the Operational Research Society. 56, 816-824. DOI: 10.1057/palgrave.jors.2601900

MAYER, R. H. e STARK, R. M. (1981) Earthmoving Logistics. Journal of the Construction Division. 107(2), 297-312. 
RICARDO, H. S. e G. CATALANI (2007) Manual Prático de Escavação - Terraplenagem e Escavação de Rocha. São Paulo: PINI. 